\title{
Un intérieur domestique traditionnel à Loches
} vers 1905

Pour une relecture ethnohistorique des premières cartes postales tourangelles

\section{Daniel Schweitz}

\section{(2) OpenEdition Journals}

Édition électronique

URL : http://journals.openedition.org/abpo/924

DOI : $10.4000 / a b p o .924$

ISBN : 978-2-7535-1501-7

ISSN : 2108-6443

\section{Éditeur}

Presses universitaires de Rennes

Édition imprimée

Date de publication : 30 mars 2006

Pagination : 141-157

ISBN : 978-2-7535-0273-4

ISSN : 0399-0826

\section{Référence électronique}

Daniel Schweitz, «Un intérieur domestique traditionnel à Loches vers 1905 », Annales de Bretagne et des Pays de l'Ouest [En ligne], 113-1 | 2006, mis en ligne le 30 mars 2008, consulté le 20 avril 2019. URL : http://journals.openedition.org/abpo/924; DOI : 10.4000/abpo.924 


\title{
Un intérieur domestique traditionnel à Loches vers 1905
}

\author{
Pour une relecture ethnohistorique \\ des premières cartes postales tourangelles
}

\author{
Daniel SchweITZ \\ Docteur en anthropologie historique
}

\begin{abstract}
De nombreuses cartes postales anciennes ${ }^{1}$ ont été publiées durant les deux dernières décennies, en Touraine comme ailleurs, mais pour l'essentiel à titre d'illustration dans des ouvrages "demi-savants ", et sans que l'on s'efforce d'en étudier véritablement le contenu en tant que source historique. Il y aurait pourtant intérêt à revisiter la série des dizaines de milliers de cartes postales éditées entre les dernières années du XIXe siècle et les années $1930^{2}$, comme à étudier celles de ces vues, pour le moins quelques milliers, qui présentent un intérêt d'ordre ethnohistorique. L'apport documentaire de ces dernières peut d'autant moins être négligé qu'elles illustrent un moment essentiel de notre histoire, celui où la société
\end{abstract}

1. Sur l'ethnohistoire et le contexte social des premières cartes postales, voir notamment : RIPERT, Aline et FRERE, Claude, La Carte postale, son histoire, sa fonction sociale, Paris/Lyon, CNRS/Presses universitaires de Lyon, 1983, 194 p.

2. De l'avis du principal marchand tourangeau, ce serait peut-être 100000 cartes postales différentes qui auraient été éditées en Touraine, dont peut-être 15000 rien qu'à Tours, dans les dernières années du XIX et au début du XXe siècle. Toutes les villes, pratiquement tous les villages, presque tous les monuments historiques ont été photographiés, mais le nombre de cartes éditées varie considérablement d'un site ou d'un monument à l'autre, selon leur intérêt touristique. Rien que pour Chenonceaux ce serait peut-être 600 cartes différentes qui auraient été publiées, plus de 400 pour le village de La Croix-en-Touraine, environ 130 pour celui de Civray-sur-Cher, mais pas plus d'une quinzaine pour celui de Beaumont-Village. Cette disparité des éditions renvoie à l'intérêt présenté par les sujets à photographier, à la motivation des différents éditeurs et des commerçants locaux, à l'estimation de ce qu'ils espéraient pouvoir écouler. Pour ces raisons, certaines vues ont été tirées à plusieurs milliers d'exemplaires : gare et rues de Tours, châteaux royaux du Val de Loire, d'autres à peut-être une cinquantaine d'exemplaires : commerces de petits villages, événements d'intérêt strictement local (" La carte postale ancienne ", Le Magazine de la Touraine, 73, 1999-2000, p. 13). Vers 1905-1907 on aurait produit annuellement, selon les estimations, de 300 à 600 millions de cartes postales en France, seulement 126 millions après 1926 pour remonter à un milliard et demi en 1959 (RIPERT, A. et FrERE, C., op. cit., 1983, p. 42-43). 
rurale " traditionnelle " va définitivement s'effacer devant les formidables mutations qui caractérisent le tournant du XIX ${ }^{\mathrm{e}}$ siècle et les premières décennies du siècle suivant.

Dès le début du $\mathrm{xx}^{\mathrm{e}}$ siècle, ces vues ont d'ailleurs été regardées comme une documentation à ne pas négliger dans les recherches portant sur les arts et traditions populaires. Arnold Van Gennep (1873-1957), dont le Manuel de Folklore français contemporain (1937-1958) marque la naissance de l'ethnologie contemporaine dans le domaine français, disposait d'une importante collection de cartes postales. Il considérait que ces vues étaient susceptibles de faire ressortir les variations des faits ethnographiques qu'il s'efforçait de décrire, par exemple dans le domaine du costume populaire. Certaines de ses vues ont été annotées par ses soins, après vérification de leur validité ethnographique, en vue de préparer une publication qui n'aboutira malheureusement pas ${ }^{3}$.

Un seul exemple suffira, ici, à mettre en lumière la richesse potentielle de cette documentation, encore mal exploitée en Touraine, celui d'une carte postale des premières années du $\mathrm{xx}^{\mathrm{e}}$ siècle représentant un couple âgé ${ }^{4}$ photographié devant son âtre, dans sa maison de la "Montée de Pointu " à Loches ${ }^{5}$, vers 1905-1910 (Figure 1). On s'efforcera notamment de cerner les évidences ethnohistoriques qui apparaissent au cours d'une relecture prenant notamment en compte les différents plans et les détails de cette carte (observés sous grossissement, après numérisation). Cette démarche de type ethnohistorique s'inspire des travaux fondateurs de Suzanne Tardieu (CNRS) et de leurs prolongements dans la pratique muséographique du musée national des Arts et Traditions populaires de GeorgesHenri Rivière ${ }^{6}$. Elle prend naturellement en compte les quelques travaux universitaires qui se sont efforcés, en Berry comme en Touraine, de suivre les pistes de recherche tracées par Suzanne Tardieu, Georges-Henri Rivière et leurs collaborateurs depuis l'après-guerre ${ }^{7}$.

3. Hier pour demain. Arts, Traditions et Patrimoine, catalogue d'exposition, Paris, Galeries nationales du Grand Palais, 13 juin- ${ }^{\mathrm{er}}$ septembre 1980, Paris, Éd. de la Réunion des musées nationaux, 1980, p. 439; " La carte postale ", Le petit journal des grandes expositions, 72, catalogue d'exposition, Paris, musée national des Arts et Traditions populaires, 23 novembre 1978-5 novembre 1979, Paris, éd. de la Réunion des musées nationaux, 1978, n.p. [p. 4].

4. Il a " 60 ans de ménage " précise la légende imprimée au bas de la carte; il appartient donc à une génération qui a connu à la fois l'épanouissement de la civilisation rurale traditionnelle vers 1850, puis l'introduction des ferments de sa destruction à l'approche de la fin du siècle.

5. Il s'agit en réalité de la rue Roche-Appert. On ignore l'origine de cette appellation de "Montée de Pointu ", qui doit être d'origine populaire puisque la rue porte déjà son nom actuel dans les recensements de la population de 1906 et 1911.

6. Voir TARDIEU, Suzanne, La Vie domestique dans le Mâconnais rural préindustriel, Paris, Institut d'ethnologie, musée de l'Homme, 1964, 539 p.; TARDIEU, S. et Hamon, Michèle, "Équipements et activités domestiques ", Guides ethnologiques du musée national des Arts et Traditions populaires, 10-11, Paris, éd. des Musées nationaux, 1972.

7. DESQUAND, Agnès, L'Équipement domestique du Sancerrois aux XIX et XX siècles : le foyer, la cuisine et l'âtre, mémoire de l'École du Louvre, Paris, 1976, dactyl., 3 vol.; ScHWEITZ, 
Figure 1

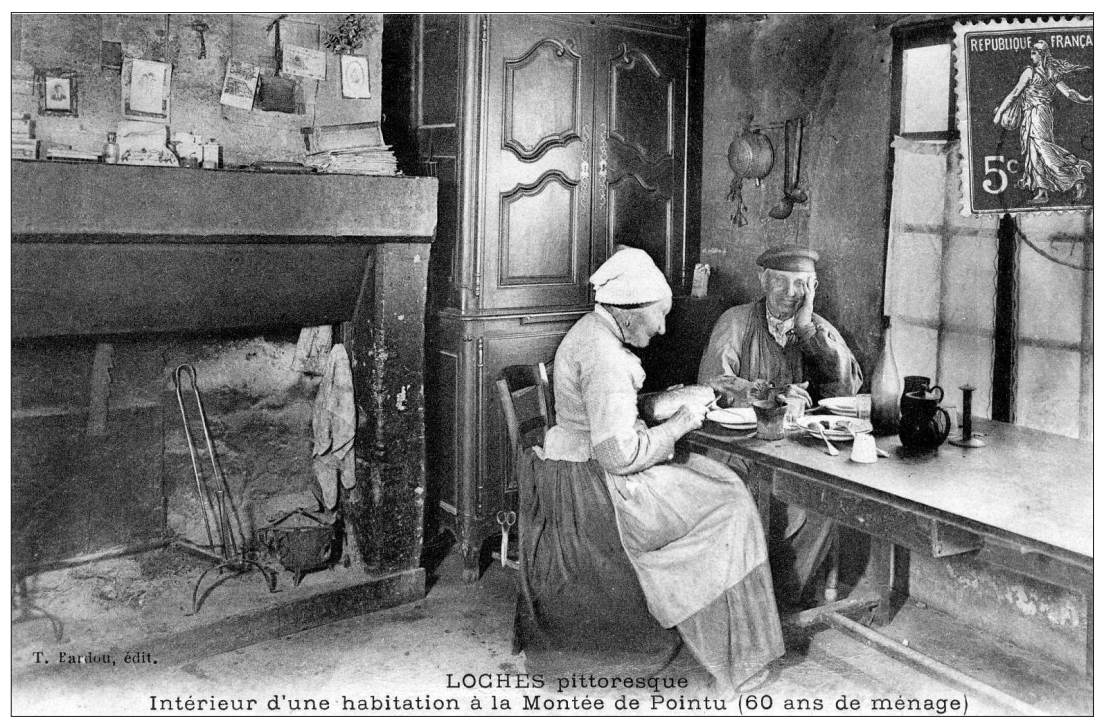

Cette carte postale faisait partie de ce qui pourrait être une petite " série " consacrée à " Loches pittoresque ", éditée vers $1905-1910^{8}$ par un commerçant de la ville : Th. Bardou. Une seconde carte de cette probable série, postée en 1915, montre le même couple âgé posant, avec un jeune homme, devant une maison de la " Montée de Pointu " (Figure 2). Cette maison, qui existe encore au numéro 18 de la rue Roche-Appert, pourrait éventuellement être celle dont la " chambre à feu " a été photographiée pour la carte qui retient ici notre attention. L'une de ses " cartes-réclames " détaille ce que vendait Th. Bardou dans son magasin de la place de l'hôtel de ville ${ }^{9}$ : "mercerie, bonneterie, chapeaux pour dames, chaussures en tous genres, corsets, lingerie, chemiserie, ganterie, parfumerie, cravates ». Les annuaires des années 1905-1910 le signalent plus simplement comme mercier, mar-

Daniel, Les ustensiles en céramique dans l'équipement domestique préindustriel du centre de la France (XIVe-début XX $X^{e}$ siècle), thèse de troisième cycle, anthropologie historique, Paris, École des Hautes Études en Sciences Sociales, 1983, dactyl., 2 vol.; ScHWEITZ, Arlette, L'Espace domestique rural en Touraine septentrionale (1850-1930). Les archives notariales et leurs apports documentaires, thèse de troisième cycle, ethnologie, Paris, École des Hautes Études en Sciences Sociales, 1984, dactyl., 2 vol.

8. Cette carte postale a été postée en 1911; une autre en 1910. Au recto, elle est divisée par un filet central séparant une partie réservée à la correspondance de celle destinée à faire figurer l'adresse, sous la mention "Carte postale ». Ce type d'organisation renvoie à une consigne donnée par l'administration en novembre 1903, pour satisfaire les collectionneurs qui désiraient préserver l'illustration figurant au verso de leurs cartes (ZEYons, Serge, Les Cartes postales, Paris, Hachette, 1979, p. 43).

9. C'est à l'intérieur de la porte Pycois, qui jouxte l'hôtel de ville et se situe devant son magasin, que seront installées en 1914 les vitrines du premier état du musée du Terroir de Loches. 
Figure 2

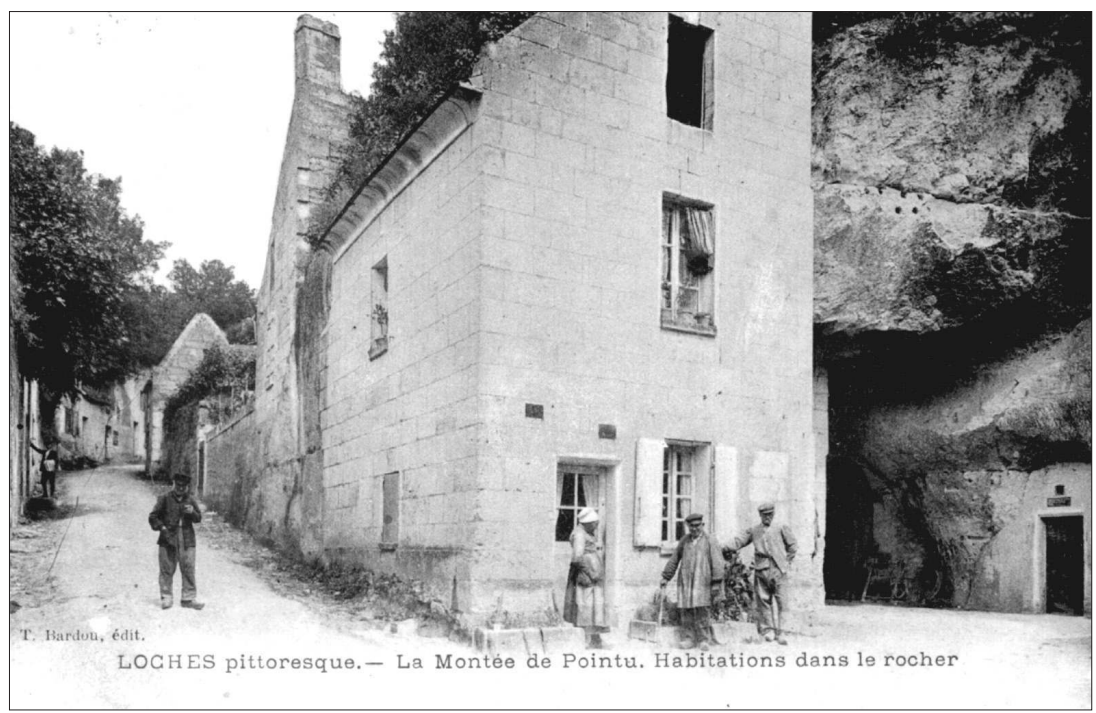

chand de chaussures et de "laines filées ", son épouse étant présentée comme " modiste ${ }^{10}$ ".

Parmi les cartes éditées par Th. Bardou dans les premières années du $\mathrm{xx}^{\mathrm{e}}$ siècle, outre des vues de la place de l'Hôtel de ville mettant naturellement en valeur son magasin (Figure 3), on remarque des vues plus pittoresques qui portent à penser qu'il a été en relation avec le "traditionniste " Jacques-Marie Rougé ou tout au moins qu'il a compris l'intérêt d'une exploitation commerciale du folklore lochois. On remarque notamment des cartes postales représentant des costumes et des coiffes traditionnels ainsi que les marchés paysans de Loches. Ces vues, mettant en valeur le pittoresque des coiffes et de différents aspects du genre de vie traditionnel à Loches, sont éditées dans un contexte où les idées régionalistes, les recherches de " traditionnistes " comme Jacques-Marie Rougé, commencent à inventer un " pays " et une " tradition lochoise " qui seront vite repris et valorisés pour les besoins d'un tourisme en plein essor ${ }^{11}$.

10. Annuaire statistique et commercial de Tours et du département d'Indre-et-Loire, Tours, Deslis, années 1905 à 1910.

11. Sur "l'invention du folklore lochois " voir ScHwErTz, Daniel, " L'œuvre ethnographique de Jacques-Marie Rougé (1873-1956) ", Bulletin de la Société archéologique de Touraine, XLII, 1990, p. 277-296; "Une source de l'identité tourangelle : le musée du Terroir de Loches ", Bulletin de la Société archéologique de Touraine, XLIII, 1992, p. 679-702; Histoire des identités de pays en Touraine (XVI -XX siècle), Paris, L'Harmattan, 2001, p. 331-346. 
Figure 3

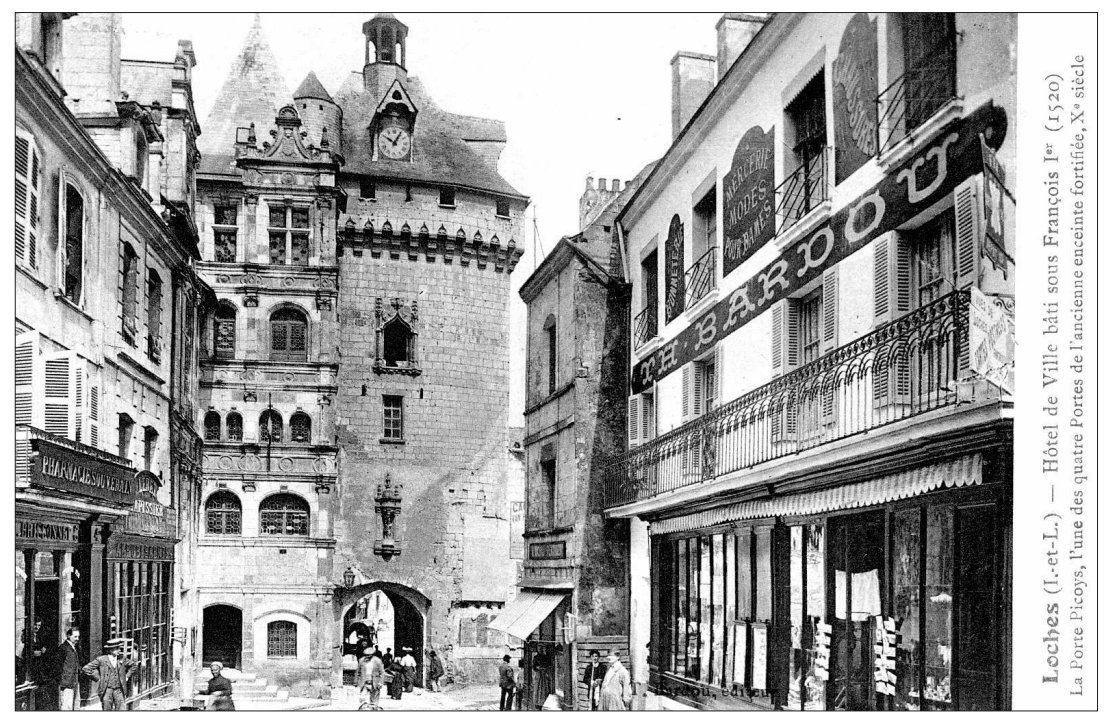

\section{Des intérieurs domestiques rarement photographiés}

La carte postale qui retient ici notre attention, à titre d'exemple, illustre ce qui représentait encore une part essentielle du quotidien de nombreuses femmes, en milieu rural, jusqu'au début du $\mathrm{Xx}^{\mathrm{e}}$ siècle : la vie et les activités culinaires autour de l'âtre domestique ${ }^{12}$. En dehors du temps des repas et des veillées, où elle s'ouvrait plus largement aux hommes, la " chambre à feu " constituait un espace essentiellement dévolu à la maîtresse de maison, éventuellement aidée de ses enfants et des parents âgés qui finissaient leur existence au coin de l'âtre.

Cette carte constitue un témoignage ethnohistorique d'autant plus précieux qu'elle appartient à un type bien moins courant que les innombrables vues représentant les monuments et les rues des villes, bourgs et villages de Touraine. Cette remarque vaut certainement pour la majorité des provinces françaises, même si certaines d'entre-elles, comme la Bretagne par exemple, peuvent dans une certaine mesure la démentir. Un certain nombre de cartes, en Touraine ${ }^{13}$ comme en Berry ${ }^{14}$, évoquaient cependant ces inté-

12. En ce qui concerne la Touraine voir SchweITz, Daniel, Cuisiner et vivre autour de l'âtre rustique en Val de Loire. Touraine, Berry, Orléanais, Anjou (XIXe-début XXe siècle), Saint-Cyrsur-Loire, Alan Sutton, 2003; SchweITz, Arlette, La Maison tourangelle au quotidien. Façons de bâtir, manières de vivre (1850-1930), Paris, Publications de la Sorbonne, 1997.

13. C'est le cas de la série des "Habitations du bord du Cher ", éditée par le Grand Bazar de Tours, ou de cartes comme : "Intérieur d'habitation dans le Rocher de Vouvray »; " En Touraine. Dentellières tourangelles".

14. Voir par exemple la fameuse série : "Les chansons de Jean Rameau illustrées ", 
rieurs domestiques, avec des photos de paysans âgés prenant la pose devant leur cheminée. Il pouvait également s'agir de médiocres reconstitutions "pour touristes", mettant en scène (dans le studio du photographe?) des acteurs posant devant un décor fait de toile et de bois peint, enrichi de quelques meubles et d'objets domestiques en rapport avec le thème choisi ${ }^{15}$.

La rareté des cartes postales représentant de véritables intérieurs domestiques résulte de plusieurs contraintes, d'ordre tant psychologique que technique et juridique. Il y a d'abord la réticence générale à laisser photographier l'intérieur d'une maison et une intimité domestique regardée comme plus ou moins présentable à un visiteur, en dressant une barrière toute symbolique mais qui s'oppose, aujourd'hui encore, à l'introduction du regard ethnographique au plus profond de cette intimité domestique ${ }^{16}$. On note cependant que dans la " chambre à feu ", la cheminée et ses abords immédiats constituaient une partie de l'espace domestique que l'on hésitait moins que d'autres à présenter aux visiteurs ${ }^{17}$, tout au moins à ceux qui étaient autorisés à dépasser le seuil de la maison où ils étaient tout d'abord accueillis. En ce qui concerne les personnes âgées sollicitées par les photographes de cartes postales, il y a certainement eu des refus nés du souci de ne pas céder au voyeurisme des touristes et des citadins, de ne pas faire figure de curiosités pittoresques, peut-être aussi de ne pas être regardés comme des "vestiges " d'un mode de vie en passe de disparaître ${ }^{18}$.

Les photographies réalisées en extérieur, devant l'entrée de la maison, de la " cave-demeurante " des troglodytes, du magasin ou de l'atelier villageois, ne présentaient pas les mêmes difficultés de cadrage et d'éclairage

présentant notamment un couple de vieux paysans ou une mère et ses filles devant leur cheminée (cartes 82, 63), ou devant leur lit (carte 79).

15. Voir par exemple : "117. Scènes du Berry ", carte éditée par Marmour à Châteauroux. Une carte éditée par Genès à Clermont-Ferrand, représentant une grossière reconstitution " d'intérieur de ferme auvergnate ", a même pour toile de fond le mur extérieur d'une église, sur lequel a été clouée une partie des objets accompagnant la fermière posant devant un âtre factice (coll. Schweitz).

16. L'enquête des chercheurs du musée national des Arts et Traditions populaires sur le " mobilier traditionnel " s'était déjà heurtée, en 1940, à la méfiance des paysans tourangeaux, dont certains refusaient de laisser photographier ne serait-ce que leurs meubles les plus intéressants (SchwerTz, A., op. cit., 1997, p. 18).

17. Voir Schweitz, D., op. cit., 2003, p. 11-24.

18. Dès les années 1920 , le regard porté sur le mobilier " rustique " va peu à peu se transformer lorsque ce dernier commencera à acquérir une valeur patrimoniale et marchande pour les citadins (Mauméné, Albert, " Maisons, jardins et meubles des bords de la Loire ", Vie à la campagne, 84, décembre 1933, notamment p. 36-51). Jacques-Marie Rougé a d'ailleurs souligné que cette évolution avait été l'un des facteurs qui l'avait amené à poursuivre ses efforts pour la création du musée du Terroir de Loches en 1925 (RougE, J.-M., Voyage en Touraine inconnue. II - À travers le Lochois [...], Tours, Imprimerie tourangelle, 1928, 155). C'est également en 1925 qu'Émile Bayard, inspecteur des Beaux-Arts, publie Les meubles rustiques régionaux de France (Paris, libr. Garnier, 1925), ouvrage de référence où il consacre un petit chapitre au mobilier exposé dans le tout nouveau musée de Loches (p. 177-187). 
que celles réalisées en intérieur. Hormis le cas où l'opérateur demandait aux occupants de poser devant leur habitation, leur magasin ou leur atelier, ces photographies réalisées dans l'espace public ne nécessitaient pas d'autorisation ${ }^{19}$. Les photographies prises à l'extérieur des habitations troglodytiques étaient ainsi plus faciles à réaliser, et satisfaisaient tout autant la demande de vues pittoresques des touristes et des consommateurs locaux. Ces dernières, même si elles ne portent pas sur l'intérieur de la maison, nous informent néanmoins sur un espace domestique qui s'étend en fait autour de cette maison, aux lieux et aux locaux qui lui sont intimement liés par des cheminements et des activités quotidiennes. Cet espace domestique extérieur, notamment dans les habitats troglodytiques les plus pittoresques, a pu faire l'objet de plusieurs photographies sous des angles différents, avec un cadrage plus ou moins large, au fil des saisons ou à plusieurs années d'intervalle. Ces séries sont particulièrement intéressantes dans la mesure où elles permettent également de repérer des transformations discrètes, liées par exemple au cycle des travaux saisonniers, et qui ne sont pas sans rapport avec les activités domestiques (jardinage, bassecour, petit élevage, lessive, etc.).

Ces quelques cartes postales du début du $\mathrm{xx}^{\mathrm{e}}$ siècle permettent d'observer ce qu'était le détail de l'organisation d'un intérieur domestique traditionnel en Touraine, réalité pour laquelle on ne dispose (apparemment) d'aucune photographie, peinture ou dessin réalisé antérieurement. Avec quelques plans de "chambres à feu " dressés par les ethnographes du musée national des Arts et Traditions populaires vers $1940^{20}$, la seule documentation disponible pour l'étude de ces intérieurs traditionnels est constituée par l'importante série des inventaires après décès rédigés par les notaires tourangeaux des $\mathrm{XVIII}^{\mathrm{e}}$ et $\mathrm{XIX}^{\mathrm{e}}$ siècles. Presque trois cents de ces inventaires après décès ont déjà été mis à profit par Arlette Schweitz pour une étude ethnohistorique portant sur l'espace et la vie domestiques en Touraine septentrionale entre 1850 et $1930^{21}$, et un bon nombre d'autres pour des études historiques décrivant l'équipement domestique du $\mathrm{XVIII}^{\mathrm{e}}$ siècle $^{22}$.

Cette documentation écrite localise et décrit avec une certaine précision les meubles et les objets présents dans la " chambre à feu ", puis dans le reste de l'espace domestique; elle permet aussi d'apercevoir des groupements d'objets dans la cheminée, les meubles (buffet, armoire, coffre,

19. Une décision du tribunal de Narbonne proclame d'ailleurs en 1905 : « le droit de vue qu'à tout individu sur tout ce qu'il y a dans la rue ".

20. Voir cinq de ces plans reproduits dans SchweITZ, A., op. cit., 1997, p. 152-153.

21. Voir SchweITZ, A., op. cit., 1997, notamment p. 25-38.

22. Voir plusieurs mémoires de maîtrise d'histoire moderne soutenus à l'université François Rabelais de Tours par : Stéphane Malmert (1991), Laurent Phamphile (1992), Corinne Boisard (1995), Valérie Fichepain (2000), Véronique Ozvalda (2000), etc. Malgré leur intérêt, ces études historiques laissent malheureusement percevoir une méconnaissance de la démarche et des travaux ethnographiques concernant l'équipement domestique traditionnel. 
maie) et les autres espaces de rangement (étagères, placard, pierre d'évier de la " bassie ", " chambre froide ", cellier). Mais il faut avoir à l'esprit que certains objets restent absents des inventaires même les plus soigneusement établis ${ }^{23}$, du fait notamment des tentatives de fraude à la succession, de l'omission des biens acquis avant le mariage ou appartenant à un tiers, mais aussi en raison de leur faible valeur ou de leur caractère religieux. D'autres objets de faible valeur, tels les ustensiles en céramique ou ceux qui sont usés ou hors d'état de servir, peuvent être regroupés pour être " prisés " en lots. Mais même les plus détaillés de ces inventaires ne précisent pas l'emplacement exact des meubles et des objets dans la " chambre à feu ", et surtout ne permettent pas de visualiser l'équipement en place, comme peut le faire une photographie suffisamment rapprochée ${ }^{24}$.

\section{Un espace domestique organisé selon la tradition}

La vue qui retient ici notre attention présente un couple âgé attablé entre cheminée et fenêtre, c'est-à-dire au cœur de sa " chambre à feu ", près des espaces fonctionnels où se déroulaient les principales activités du quotidien domestique ${ }^{25}$. Le cadrage de cette photographie met d'ailleurs en valeur deux images clairement reconnues de la "Vieille Touraine " au début du $\mathrm{XX}^{\mathrm{e}}$ siècle $^{26}$ : la cheminée et son âtre, regardés comme les symboles de la vie domestique et familiale, une femme en coiffe et un homme en blouse, constituant une parfaite illustration de l'habillement ordinaire des campagnards du XIX ${ }^{\mathrm{e}}$ siècle. La photographie de ce couple âgé devant son foyer comporte une forte charge symbolique. Elle évoque le souvenir d'une époque, guère lointaine pour de nombreux citadins du début du $\mathrm{xx}^{\mathrm{e}}$ siècle, où les parents âgés venaient finir leurs jours près de l'âtre rustique, où les veillées en faisaient un lieu de convivialité, de transmission des savoir-faire et des traditions, parfois aussi de rapprochements avec les filles à marier ${ }^{27}$. Elle rappelle aussi la pratique des inventaires après décès, où le notaire décrivait tous les meubles et objets présents dans une maison en com-

23. Voir SchweITZ, A., op. cit., 1997, p. 35-38. Ces inventaires correspondent en outre à des situations particulières, comme le souci de protéger les intérêts des enfants mineurs, et ils ne sont pas nombreux lorsque l'on considère la série des actes dressés, année après année, par chacune des études tourangelles.

24. Ils ne permettent pas de repérer les meubles qui servent, au moins symboliquement, à diviser l'espace de la " chambre à feu ". C'est le cas des chaises alignées le long du lit, qui pourraient marquer une séparation entre espace diurne et nocturne (SCHWEITZ, A., op. cit., 1997, p. 125), selon une disposition que l'on observe sur au moins trois vues d'intérieurs troglodytiques tourangeaux vers 1900-1910 (coll. Schweitz).

25. Voir SchweITZ, D., op. cit., 2003, p. 33-40.

26. Elles étaient regardées avec condescendance par les modernistes et la jeune génération, mais néanmoins valorisées par tous ceux qui s'attachaient à la sauvegarde des traditions locales (folkloristes, régionalistes, "petits patriotes "), ou à les instrumentaliser pour favoriser un tourisme en passe de devenir une activité économique notable (commerçants, élus locaux, acteurs des premiers syndicats d'initiative, fondateurs des musées du terroir).

27. Voir SchweiTz, D., op. cit., 2003, p. 14-24. 
mençant par l'intérieur de la cheminée, dans la " chambre à feu ", selon une pratique constatée en Touraine comme ailleurs jusqu'au début du Xx ${ }^{\mathrm{e}}$ siècle.

Dans la cheminée, qui constituait une source de chaleur durant la mauvaise saison et un éclairage complémentaire, on effectuait la préparation et surtout la cuisson des aliments, on chauffait l'eau nécessaire aux ablutions et aux travaux domestiques. Celle qui apparaît sur la photographie appartient à un type très commun au XIX ${ }^{\mathrm{e}}$ siècle, avec un manteau peu saillant monté sur de forts jambages quadrangulaires, avec surtout un retrait permettant de laisser la place d'une étroite tablette au-dessus de son linteau. Une large planche est fixée en travers de l'ouverture de la cheminée, sous ce linteau, afin de rabattre vers l'intérieur du foyer une partie des fumées qui pouvaient s'échapper dans la " chambre à feu ". C'est également pour réduire cet inconvénient que le sol de l'âtre a été installé un peu plus haut que le carrelage de la salle. Cette cheminée constituait un espace de rangement pour une partie de l'équipement culinaire et divers objets usuels, qui pouvaient être placés dans un angle de l'âtre, suspendus à des pitons sur les parois ou sur les jambages du foyer, déposés sur la tablette ou fixés sur le devant du manteau.

La table est repoussée contre la fenêtre pour fournir un meilleur éclairage aux convives, position probablement mieux adaptée à l'usage d'un couple âgé, peut être accompagné d'un parent ou d'un employé (vu le nombre de couverts présents). Mais cette position pourrait éventuellement avoir été réclamée par le photographe désirant obtenir une meilleure luminosité pour son travail. Les principaux repas, hors ceux pris "sur le pouce " à l'extérieur, étaient consommés à cette table, devant la fenêtre qui fournissait non seulement un éclairage pour les travaux de couture de la maîtresse de maison, mais qui lui permettait aussi de surveiller la cour au travers de la transparence des rideaux. Cette table marquait plus ou moins la séparation, toute symbolique, entre l'espace de cuisson qu'était la cheminée et l'espace de préparation des aliments et de petits lavages constitué par la pierre d'évier. Pour débarrasser cette table, les couverts et les autres objets indésirables entre les repas pouvaient être placés sur une autre petite table, sur la tablette de la cheminée ou sur l'entablement de la fenêtre.

Le buffet deux-corps situé en arrière-plan, entre le montant de la cheminée et l'angle du mur, est un meuble de rangement où vient normalement prendre place de la vaisselle (assiettes, plats, soupière, verres, pichets), peut-être aussi des couverts (cuillers, fourchettes, couteaux) si elle est pourvue d'un tiroir. Ce beau meuble, appartenant à un type qui a été couramment fabriqué par les menuisiers tourangeaux de la seconde moitié du XIX ${ }^{\mathrm{e}}$ siècle, devait être remarqué dans la " chambre à feu " avec ses élégantes moulures de style Louis XV et ses ferrures en acier poli. Sa présence dans la pièce à vivre pouvait d'ailleurs être regardée, au moins à l'origine, et s'il ne s'agit pas déjà d'une pièce acquise auprès d'un brocanteur, comme la marque d'un certain statut social. 
On notera que la prise de vue ne montre pas la totalité de la " chambre à feu " et qu'elle laisse notamment de coté des espaces fonctionnels aussi importants que la «bassie " et sa pierre d'évier, la porte, le ou les lits. Dans la " bassie " et sur sa pierre d'évier, généralement situés près de la porte, souvent aussi dans l'embrasure d'une fenêtre, on logeait le seau contenant l'eau tirée du puits. Des étagères ou des placards permettaient d'y ranger certains ustensiles culinaires. On y lavait et on y préparait les aliments, on y effectuait de sommaires ablutions. La porte, dont le battant supérieur était le plus souvent laissé ouvert pour aérer une salle plus ou moins enfumée par la cheminée, était le lieu où s'effectuaient des travaux domestiques demandant un meilleur éclairage. C'était naturellement l'endroit où le contact était pris avec les visiteurs, avant de les inviter à pénétrer dans la " chambre à feu ", jusque devant la flambée allumée dans l'âtre pendant la saison froide. Le lit ou les lits, qui pouvaient accueillir un malade ou un vieillard, étaient généralement placés sur l'un des côtés de la cheminée, afin de bénéficier de la chaleur d'un foyer qui était allumé dès l'aube par la maîtresse de maison. On cherchait à les loger de préférence dans la partie la plus sombre de la " chambre à feu ", loin de la fenêtre et de la porte où venaient s'engouffrer des courants d'air froid.

Parmi les meubles d'usage commun susceptibles d'être également présents dans cette " chambre à feu ", hors du champ de la photographie, on peut citer la maie (ou pétrin), l'armoire, peut-être le buffet ${ }^{28}$. Une maie, inutilisée pour pétrir le pain domestique depuis qu'il était acheté au boulanger, pouvait renfermer les miches de pain et des provisions de bouche, telles que du lard, des rillettes, du fromage et, éventuellement, les reliefs d'un précédent repas. Comme dans le placard ou sur les étagères de la "bassie ", il était également possible d'y ranger de la vaisselle d'usage courant et des ustensiles culinaires. Une armoire pouvait abriter du linge de maison, des vêtements et des papiers importants. Le buffet bas (ou « basset "), qui aurait fait ici double usage avec le buffet à deux corps, aurait pu abriter de la vaisselle sur ses étagères et des couverts dans ses tiroirs.

On peut naturellement se poser la question de savoir à quelle catégorie sociale et à quel type d'habitat rattacher cet espace domestique. L'aspect général de la " chambre à feu ", la nature des vêtements portés par le maître de maison et son épouse, comme par ailleurs la nature de la population qui demeure rue Roche-Appert au début du Xx ${ }^{\mathrm{e}}$ siècle ${ }^{29}$, portent à penser qu'il s'agit d'une catégorie sociale plutôt modeste. Le maître de maison n'était vraisemblablement pas un journalier, certainement pas un cultivateur, mais plus probablement un (ancien) artisan, marchand ou ouvrier

28. Voir SchweITZ, A., op. cit., 1997, p. 236-243.

29. Les recensements du début du $x^{\mathrm{e}}$ siècle montrent que les journaliers, journalières et couturières y sont nombreux, à côté de tout un petit peuple exerçant divers métiers : blanchisseuse, boulanger, charron, cordonnier, horticulteur, jardinier, lingère, maçon, marchand de charbon, maréchal, menuisier, paveur, peintre, puisatier, sellier, etc. (Arch. dép. d'Indre-et-Loire, recensement de la population, Loches, 1896-1911, 2 MI 347/4). 
de métier. La localisation de cet espace domestique, dans une rue située aux marges déjà rurales de la ville et sous-préfecture de Loches (5000 habitants à l'époque), renvoie en tout cas à un milieu où s'interpénétraient civilisation rurale traditionnelle et civilisation urbaine.

\section{Des objets, des détails à ne pas négliger}

Dans le foyer ${ }^{30}$ on remarque une grande plaque de cheminée, une large " crémaillère à dents ", une paire de chenets en fer d'un modèle classique à l'époque, une grande " pince à feu ". Une " barre à feu ", placée en travers des chenets, paraît avoir été repoussée vers le fond de l'âtre, tout contre la plaque. Des chiffons sont pendus à des clous fixés sur le côté de l'âtre. Ils étaient destinés à saisir des ustensiles métalliques trop chauds pour être directement empoignés, par exemple l'anse ou le couvercle d'une marmite placée au-dessus du feu. Ils permettaient également de ne pas trop se salir les mains avec la suie déposée sur les ustensiles placés dans le foyer.

On note sur le côté du foyer la présence d'un petit réchaud à braises ou " cagnard ", en fer ou en fonte. Ce type de " cagnard ", qui affecte la forme d'une petite marmite, est pourvu d'une grille pour supporter les braises dont on l'emplit et d'évents, percés au-dessous du niveau de cette grille, pour activer leur combustion. Il est ici surmonté d'un " triangle ", petit trépied destiné à porter un plat ou un autre petit récipient à cuire au-dessus des braises d'un réchaud, peut-être aussi d'une brique qui sera chauffée sur ces braises avant d'être utilisée pour réchauffer le lit du couple. Ce type de réchaud à braises, d'un usage généralisé, était plutôt utilisé pour une cuisson mijotée des " petites cuisines " ou pour réchauffer les restes d'un repas. Un petit gril en fer est peut-être fixé à un clou sur le montant du buffet deux-corps, s'il n'est pas tout simplement coincé entre ce meuble et la tablette de la cheminée. Ce petit gril, utilisé sur le réchaud à braises ou sur des braises à l'avant du foyer, servait notamment à griller le hareng consommé au " petit déjeuner".

Dans ce foyer on n'observe pas de feu, de tison ou même de cendre, pas de marmite ou de poêle. On peut donc se poser la question de son utilisation pour la cuisson des aliments, car il n'est pas impossible qu'ait existé sur le côté de la cheminée (hors du champ de la photographie) un petit fourneau dont le conduit aurait été relié latéralement à son manteau. On note aussi l'absence, tout au moins dans le champ de la prise de vue, d'ustensiles ordinairement utilisés dans l'âtre : canon de vieux fusil ou soufflet employé pour attiser le feu, " pelle à feu " pour recueillir des braises, simple aile de volaille ou balayette pour nettoyer les abords de l'âtre, "servante " fixée à la crémaillère pour porter la poêle, marmite pour cuire la soupe quotidienne.

30. Sur l'équipement du foyer traditionnel en Touraine voir SchwEITZ, D., op. cit., 2003, p. 49-60; également Tardieu, S., op. cit., 1964, p. 75-82. 
Sur la tablette de la cheminée on observe par contre un alignement de petits objets, rangés ici à demeure en attendant de servir ou posés pour un instant. On y distingue une série de petites boîtes de formes et de tailles diverses, et dont certaines affectent la forme d'un pilulier, ainsi qu'une petite bouteille. De grandes boîtes sont disposées presque symétriquement sur les côtés de la tablette, avec au centre un objet ou bibelot décoré (en faïence?), d'usage indéterminé, peut-être utilisé pour caler des papiers contre le manteau de la cheminée. L'une des grandes boîtes est posée, à droite, sur une pile de papiers ou de journaux soigneusement empilés. Un verre à pied, retourné pour être protégé de la poussière et des cendres de la cheminée, est placé près de boîtes contenant peut-être des médicaments.

Outre ces éventuels médicaments, il est difficile de savoir ce qui était contenu dans ces boîtes, mais on peut songer à des allumettes et peut-être encore à des " chènevottes ${ }^{31}$ " pour allumer le feu, au nécessaire de couture, comme à des " bricoles " renvoyant à l'usage de conserver tout ce qui était susceptible de resservir. La petite bouteille, si elle ne contient pas une quelconque potion, pourrait renfermer l'eau bénite destinée, avec le concours des images pieuses fixées sur le manteau de la cheminée, à préserver la maison de la foudre et de tous les autres dangers potentiels.

Sur le manteau de la cheminée on remarque la présence de plusieurs photographies et imprimés de tailles différentes, selon une pratique commune et que l'on retrouve jusque dans les collages opérés traditionnellement sur la face intérieure des portes d'armoires. L'une des photographies (peut-être une page de calendrier), fixée par l'un de ses côtés, représente l'hôtel de ville de Loches (devant lequel se trouve le magasin de Th. Bardou). On remarque surtout la photographie d'un jeune homme cravaté, certainement un fils ou un parent, placé dans un cadre (de bois) sculpté; une gravure représentant une vierge à l'enfant, dans un encadrement derrière lequel sont passées des branches de buis ou un petit bouquet de fleurs. On observe également d'autres images, certainement de moindre importance puisqu'elles se recouvrent sur ce manteau. Deux objets sont pendus à des clous : probablement un tire-bouchon et ce qui paraît être une petite pelle. Cette dernière est attachée à un lacet suspendu à un clou, lui-même planté dans une toile ou un grand imprimé appliqué sur le manteau de la cheminée.

La table est pourvue d'un large tiroir, qui pouvait notamment être utilisé pour ranger les cuillers, les fourchettes et les couteaux, éventuellement du petit outillage employé pour des bricolages domestiques. À même le bois de cette table sont disposés trois assiettes creuses, du type de celles en usage pour consommer de la soupe, des cuillers (en étain), trois larges

31. Les vraies " chènevottes " étaient constituées de résidus du " teillage " du chanvre dont l'extrémité avait été trempée dans du souffre fondu. Elles étaient surtout utilisées pour transmettre la flamme du foyer vers un autre petit foyer à allumer (dans un " cagnard " ou un " potager ") ou vers la mèche d'un ustensile d'éclairage (TARDIEU, S., op. cit., 1964, p. 74). 
verres, une bouteille (de vin), deux pichets en terre cuite émaillée de couleur sombre, l'un pourvu d'un couvercle, pour l'eau ou une quelconque "bouette " ou " boisson " familiale, enfin un récipient d'usage indéterminé renfermant probablement une cuiller. On aperçoit également un chandelier à tirette en tôle (de fer ou de cuivre), d'un modèle assez commun à l'époque, destiné à éclairer le repas du soir et les veillées à table. Sur le dossier de la chaise est également attaché un lacet, lui-même fixé à une paire de ciseaux de dimensions respectables, probablement utilisée pour des travaux de couture sous l'éclairage de la fenêtre. Comme c'est apparemment le cas en Touraine ${ }^{32}$, c'est la maîtresse de maison qui tranche le pain avec son couteau de cuisine pour ensuite le distribuer aux convives.

L'homme porte le costume traditionnel des ruraux tourangeaux de la seconde moitié du XIX ${ }^{\mathrm{e}}$ siècle $^{33}$, tel qu'on l'observe encore sur les cartes postales montrant l'animation des marchés paysans de Loches vers 1900. Il est vêtu d'une chemise (en toile) boutonnée, sans col, et d'une blouse ample ou "biaude" (en grosse toile de couleur bleue), dont les manches sont serrées et boutonnées aux poignets. Il est coiffé d'une casquette en drap d'un type commun dans la région de Loches. Sur la photographie prise devant la maison, on remarque que l'ample blouse de l'homme descend jusqu'à mi-cuisses, sur un pantalon de couleur foncée (fait de grosse toile ou de velours); on note également qu'il est chaussé de gros sabots (probablement en noyer) (Figure 2). Sur cette dernière photographie, on remarque un autre homme, peut-être un fils ou un ouvrier, qui pourrait fort bien être le convive dont on aperçoit le couvert sur la table de la " chambre à feu ". Comme il est vêtu de façon moins pittoresque, avec un bleu de travail et une casquette ordinaire d'ouvrier, il se pourrait qu'il ait été écarté de la photographie prise en fin de repas, à l'intérieur de la maison.

L'épouse est vêtue d'une large jupe plissée de couleur foncée, sur laquelle elle a passé un tablier long, lui-même apparemment recouvert d'un tablier plus court, et de couleur moins sombre, pourvu d'au moins une grande poche fourre-tout. Ce tablier est serré à la taille, sur un caraco de couleur claire, pourvu d'une large pièce au coude. Sous ce caraco apparaît ce qui semble être le col plissé d'une chemise de corps. Cette femme est coiffée d'un bonnet tourangeau (lochois?) de type ancien, avec une large lite paillée et un fond retombant certainement orné d'une modeste broderie $^{34}$. Elle porte ce bonnet à l'ancienne mode, c'est-à-dire placé bas sur le front et couvrant bien ses cheveux ${ }^{35}$.

32. Schweitz, A., op. cit., 1997, p. 207.

33. Voir par ex. Rouge, Jacques-Marie, Le Folklore de la Touraine, Tours, Arrault, 1931, p. 21-22, fig. 10 et 12 .

34. Sous le bonnet apparaît un liseré foncé, dont on ne peut déterminer s'il s'agit d'une "sous-coiffe" en tissu noir, comme on en portait ordinairement pour faire ressortir ses broderies, ou des cheveux teints et coiffés en deux bandeaux, selon la mode du temps (Rouge, J.-M., op. cit., 1931, p. 15, note 1 et 2).

35. À l'inverse des filles et des jeunes femmes qui portent dorénavant leur bonnet très en arrière, descendu dans le cou, afin d'en faire valoir les broderies fines et de dégager 
Sur ce qui paraît être une tablette du buffet deux-corps, à portée de main derrière la chaise du maître de maison, est posé un paquet qui pourrait renfermer du tabac. Entre ce meuble et la fenêtre, un tasseau cloué au mur sert à accrocher des ustensiles de cuisine : une louche, une écumoire et ce qui paraît être une passoire, ainsi qu'un végétal indéterminé (peutêtre de la menthe?) qui pourrait avoir été utilisé pour l'assaisonnement des aliments. Ce système d'accrochage était commun sur les parois et les jambages des cheminées rurales, comme sur les solives du plafond autour de l'âtre.

La réalité domestique qui figure dans cette vue ne paraît pas avoir été arrangée par le photographe, hormis peut-être une vaisselle trop rassemblée sur la table, vraisemblablement pour figurer entièrement dans le champ de la prise de vue, et le retrait probable d'un convive correspondant au troisième couvert. Il s'agit d'une photographie prise à la fin d'un repas quotidien, probablement un "goûter " consommé aux approches de midi ${ }^{36}$. Le sourire du vieux Lochois et son attitude détendue, à une époque où les photographies de mariage montrent encore de vieux paysans visiblement embarrassés devant l'objectif, indiquent que la prise de vue a été bien acceptée. Il est vrai qu'à Loches l'exemple du "César lochois ", modeste figure de la vie populaire locale ${ }^{37}$ figurant sur plusieurs des cartes postales représentant des monuments ou des rues de la ville vers 19001910, constituait un exemple qui pouvait décomplexer les autres acteurs du pittoresque local.

\section{Un regard sur les mœurs domestiques}

Au-delà des éléments les plus directement observables : aménagements de l'espace domestique, meubles et objets, vêtements, regroupements et rangements de ces derniers, ce genre de carte postale peut également porter témoignage sur les mœurs domestiques traditionnelles, pour autant que nous soyons en mesure de passer de l'observation des caractéristiques de la vie matérielle à la perception de réalités nettement moins évidentes à définir d'après une simple photographie. On peut néanmoins s'y efforcer en replaçant cette documentation figurée dans son contexte ethnohistorique, plus ou moins connu à travers d'autres types de documents. Dans l'exemple ici retenu, on peut par exemple noter que la portion d'espace domestique représentée paraît bien tenue par la maîtresse de maison. En cela aussi, l'intérieur domestique photographié à Loches semble représentatif d'une réalité qui s'impose peu à peu dès la seconde moitié du

leurs cheveux sur le front (SchwEITZ, Daniel, La Coiffe et son imaginaire dans le folklore de la Touraine, Saint-Cyr-sur-Loire, Alan Sutton, 2002, p. 19).

36. Principal repas de la journée, on y consommait de la soupe, de la viande de porc ou du lard avec des légumes, de la charcuterie en hiver.

37. Voir SchwEITZ, Daniel, «Figures pittoresques de l'archéologie tourangelle vers 1900 : César et Léon Girardin, explorateurs des souterrains du château de Loches ", Le Val de l'Indre, 4, 1992, p. 79-84. 
$\mathrm{XIX}^{\mathrm{e}}$ siècle dans les provinces ligériennes, avec des retards certainement dans les pays les plus retardés, comme la Brenne ou la Sologne.

Henri Baudrillart, cherchant à définir les mœurs des populations agricoles de la France, a souligné en 1888 que « le paysan tourangeau a toujours aimé son chez soi " et que " ce goût n'a fait que s'accroître et se répandre ", qu'il " soigne sa maison avec une sorte de passion ". Il se dit même impressionné par une "propreté domestique qui est [...] l'une des qualités et l'un des principaux traits des campagnes de la Touraine ", et met en relation cette dernière avec les "qualités d'ordre et d'activité " des "fermières ou maîtresses " tourangelles ${ }^{38}$. Cette impression de propreté avait déjà saisi le notaire lochois François-Léonide Archambault décrivant les paysans de sa région, dans un manuscrit rédigé entre 1875 et 1880 . Il souscrivait au texte d'un petit opuscule paru à Tours en 1873, qui soulignait à la fois l'encombrement des " chambres à feu ", le fouillis régnant dans la " chambre froide " qui leur servait de débarras, mais également les efforts de la maîtresse de maison tourangelle pour garder son intérieur propre. Dans la maison paysanne de la fin du XIX ${ }^{\mathrm{e}}$ siècle écrivent ces auteurs : "Les meubles brillent sous les frictions assidues des ménagères. On se mire dans le panneau de l'armoire, le carreau est balayé tous les jours ${ }^{39}$."

Cette propreté domestique "minutieuse " caractérisait également le Berry, et George Sand, aujourd'hui regardée comme une " pionnière de l'ethnographie " dans sa province ${ }^{40}$, a souvent insisté sur ce trait des mœurs paysannes dans ses romans rustiques : Le Meunier d'Angibault (1845), La Mare au Diable (1846), Promenades autour d'un village (1847), etc. Pour l'auteur de La Mare au Diable, cette propreté domestique constitue même « la dignité du pauvre, c'est par elle qu'il se montre supérieur à sa destinée ${ }^{41}$ ". Les logements des ouvriers urbains les plus démunis et marginalisés pouvaient cependant être de véritables taudis, caractérisés par leur malpropreté repoussante et le délabrement de leur mobilier, comme c'était le cas dans les vieux quartiers de Tours vers $1850^{42}$.

La propreté qui caractérisait apparemment beaucoup d'intérieurs rustiques en Touraine pouvait également s'accommoder d'extérieurs où s'entassaient les immondices, renforçant ainsi l'opposition de plus en plus marquée entre un intérieur maintenu propre par la maîtresse de maison, et un

38. Baudrillart, Henri, Les Populations agricoles de la France. $2^{e}$ série : Maine, Anjou, Touraine [...], Genève, Mégariotis Reprints, 1978 (rééd. de Paris, 1888), p. 148.

39. Les paysans. Seizième lettre, partiellement éditée dans ScHWEITZ, A., op. cit., 1984, p. 389-390.

40. Bernard, Daniel, Georges Sand, pionnière de l'Ethnographie, s.l., chez l'auteur, 2004, p. 37 sqq.

41. Vincent, Marie-Louise, Le Berry dans l'œuvre de George Sand, Marseille, Laffitte Reprints, 1978 (rééd. de Paris, 1919), p. 134-135. Dans La Mare au Diable, par exemple, Marie nettoie et frotte tant et si bien ses pauvres meubles " quelle [les] rends clairs comme des miroirs" (chap. V).

42. ScHwEITZ, Daniel, " Vivre à Tours naguère ", dans Tours, Paris, Bonneton, 1992. p. 181, d'après les Recherches [...] sur l'hygiène de la ville de Tours (1853) du docteur Alexis Giraudet. 
extérieur où s'effectuaient des travaux sales, dans la cour, la basse-cour, le jardin et les bâtiments agricoles ${ }^{43}$. Marie-Louise Vincent, biographe de George Sand, qui est entrée vers 1900 dans " nombre de maisons " berrichonnes (de la Vallée noire), a cependant noté que même celles qui étaient habitées par des " hommes veufs ou de vieux garçons ", ne présentaient " aucun désordre extérieur ${ }^{44}$ ".

À la lumière de cette étude ponctuelle, on perçoit que certaines des cartes postales « tourangelles " éditées autour des années 1900 constituent bien une documentation historique susceptible d'être soumise à une relecture de type ethnographique. Dans l'exemple ici retenu, on constate notamment qu'elles permettent d'étudier de visu des exemples d'équipements domestiques datés, dont on peut déterminer (avec les réserves précédemment évoquées) la nature, la répartition et la disposition dans l'espace de la " chambre à feu " traditionnelle. Avec une analyse plus affinée et le secours des travaux ethnographiques, cette relecture peut même aller jusqu'à donner une idée des aires fonctionnelles et symboliques de la vie domestique. Ces vues permettent également d'observer certains détails du costume populaire, dont on ne conserve pratiquement rien dans les musées tourangeaux, comme des attitudes qui ne relèvent pas toujours d'un simple jeu d'acteur devant l'objectif du photographe.

Plus généralement, les cartes postales des années 1900 illustrent, tout au moins lorsqu'elles ne sont pas de simples mises en scène, une civilisation rurale " traditionnelle " tout à la fois proche et lointaine. Proche parce qu'elle n'a définitivement disparu que dans la première moitié du xx siècle, sous nos yeux donc, mais également lointaine parce qu'inscrite dans une " longue durée " qui plongeait ses racines jusque dans la reconstruction des campagnes tourangelles, entre le milieu du $\mathrm{Xv}^{\mathrm{e}}$ siècle et le début du $\mathrm{XVI}^{\mathrm{e}}$ siècle ${ }^{45}$. Cette civilisation rurale et ses traditions, négligées par les érudits tourangeaux de la fin du XIX ${ }^{\mathrm{e}}$ et du début du XX⿳亠丷厂犬 siècle $^{46}$, parce qu'elles relevaient du populaire et du quotidien, constitue aujourd'hui un champ de recherches où beaucoup, pour ne pas dire quasiment tout en certains domaines, reste à faire.

43. SchweITZ, A., op. cit., 1997, p. 81, 100-101, 109.

44. VINCENT, M.-L., op. cit., 1978, note 2, p. 135.

45. C'est à ce moment qu'apparaissent les cheminées murales dans les maisons paysannes ordinaires, comme l'équipement domestique qui y sera utilisé jusqu'au cours du xxe siècle (voir SchweITz, D., 2003, p. 44-46). C'est par exemple le cas des ustensiles en céramique utilisés dans l'âtre traditionnel, qui fixent leurs caractéristiques essentielles au bas Moyen Âge, en Touraine comme ailleurs (ScHWEITZ, D., op. cit., 1983).

46. Hormis quelques fortes personnalités qui n'ont pas hésité à sortir des sentiers battus de l'histoire événementielle et de l'archéologie monumentale. À partir des années 1890-1910, c'est notamment le cas de Raphaël Blanchard, Adolphe Darmezin, Léon Pineau ou Jacques-Marie Rougé, en ce qui concerne les traditions populaires, de Casimir Chevalier, ou Auguste Chauvigné, pour la civilisation paysanne. 


\section{RESUME}

De nombreuses cartes postales anciennes ont été publiées, en Touraine comme ailleurs, sans que l'on s'efforce véritablement d'en étudier le contenu en tant que source de nature à la fois historique et ethnographique.

Il y aurait pourtant intérêt à revisiter la série des dizaines de milliers de vues éditées entre 1900 et $\mathbf{1 9 3 0}$. L'apport de ces dernières peut d'autant moins être négligé qu'elles illustrent un moment essentiel de notre histoire, celui où la société rurale " traditionnelle " va définitivement s'effacer devant notre modernité.

Un seul exemple suffira à mettre en lumière la richesse potentielle de cette documentation, celui d'une carte postale des années 1900 représentant un couple de tourangeaux photographié dans son intérieur rustique. On s'efforcera de repérer et d'analyser les évidences qui apparaissent au cours d'une relecture de type ethnographique, prenant notamment en compte les différents plans et les détails de cette vue.

\section{ABSTRACT}

Many old postcards have been published, in Touraine and elsewhere, but no one ever tried to study their content as a historical and ethnographical source.

However, it would be quite interesting to reconsider the set of thousands of photographical views published between 1900 and 1930. Their utmost interest lies in the fact that they illustrate an essential moment in our history, the moment when traditional rural society definitely gave way to our modem way of life.

A single example will bring out the potential fullness of the information; a postcard from the 1900s and showing a couple in their rustic Touraine interior. We will try to find and analyze the obvious facts which appear after a second reading of ethnographical type, taking particularly into account the different levels and details of this photo. 